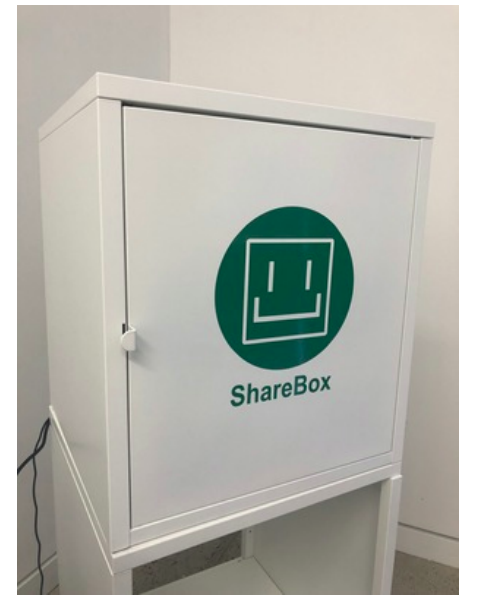

Figure 1: ShareBox-a platform for borrowing and lending items in local communities-at one deployment site.

\title{
Challenges and Lessons Deploying a Physical System for Resource Exchange in Local Communities
}

\author{
Andrea Gallardo \\ Cornell Tech
}

\author{
Nicola Dell, Mor Naaman \\ The Jacobs Institute, Cornell Tech
}

\section{ABSTRACT}

This interactive poster will discuss challenges and lessons learned designing and deploying ShareBox, a hardware-based system that enables people to share physical resources within local communities. Our goal in sharing the insights and struggles we encountered creating ShareBox is to help other researchers working on similar platforms to avoid the pitfalls that impacted our research.

\section{CCS CONCEPTS}

- Human-centered computing $\rightarrow$ Field studies.

\section{KEYWORDS}

Indirect resource exchange; sharing; sharing economy

\section{ACM Reference Format:}

Andrea Gallardo and Nicola Dell, Mor Naaman. 2019. Challenges and Lessons Deploying a Physical System for Resource Exchange in Local Communities. In 2019 Computer Supported Cooperative Work and Social Computing Companion Publication (CSCW '19 Companion), November 9-13, 2019, Austin, TX, USA. ACM, New York, NY, USA, 5 pages. https://doi.org/10.1145/3311957.3359497

Permission to make digital or hard copies of part or all of this work for personal or classroom use is granted without fee provided that copies are not made or distributed for profit or commercial advantage and that copies bear this notice and the full citation on the first page. Copyrights for third-party components of this work must be honored. For all other uses, contact the owner/author(s).

CSCW'19 Companion, November 9-13, 2019, Austin, TX, USA

(c) 2019 Copyright held by the owner/author(s).

ACM ISBN 978-1-4503-6692-2/19/11.

https://doi.org/10.1145/3311957.3359497 


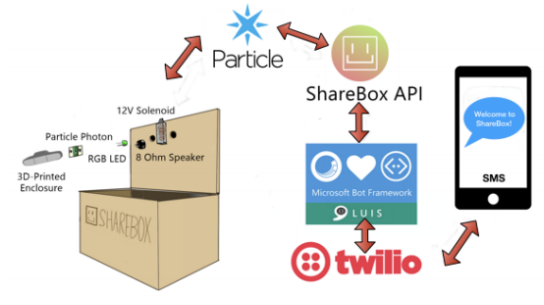

Figure 2: ShareBox is a physical locker controlled by an SMS-driven chatbot. The lock is a simple solenoid interfaced via a Particle Photon through the Particle Cloud API, and the chatbot is built on $\mathrm{Mi}$ crosoft's Bot Framework, integrated with their LUIS parser and Twilio's SMS API. The ShareBox API exposes user, inventory, and transaction information. [5]

\section{INTRODUCTION}

We further developed a peer-to-peer exchange model to allow anonymous and asynchronous sharing in local communities. Deployment efforts were unsuccessful, as explained below.

CSCW's interest in resource exchange is growing [1, 3, 4, 6, 7]. As researchers, we were interested in Indirect Resource Exchange, a generalized form of sharing that does not require reciprocity; primarily peer to peer transactions that benefit one party. Our physical system, ShareBox, was designed to facilitate sharing among community members. We hope that researchers and designers interested in designing shared physical systems will find our insights valuable.

\section{What is ShareBox?}

ShareBox (shown in Figure 1) is a physical, remote-controlled locker that enables people to exchange items with members of their community. Through the website, borrowers and lenders can sign up, as well as browse and list items. The locker provides a space where participants can drop off items to be lent or pick up items to be borrowed, and the coordination and interaction with the box is done via a text messaging chatbot.

The current iteration of ShareBox was deployed from February 2019 to June 2019 and located on the Cornell Tech campus, in a common area for Master's degree students. The population of 24 users who signed up to use ShareBox was comprised of nearly all Master's degree students, with the exception of a PhD student, a staff member and a ShareBox researcher.

\section{SHAREBOX RESEARCH METHODS}

Outreach: Before deploying the current iteration of ShareBox, the team reached out to several local organizations that already seemed to have a sense of community in which members trust each other We developed relationships online and offline, doing in-person outreach in Astoria, Queens, Brooklyn, and Manhattan. While we were not successful in deploying at external sites, we were able to gather some of their concerns, which will be discussed in the "Challenges" section.

During the deployment, we conducted outreach through in-person events, flyers, emails to listservs, and messages to a student Slack group.

Interviews with Potential Participants Prior to deployment, we conducted five unofficial but structured interviews. Based on these, we purchased and listed certain items available for borrowing on the ShareBox website, in order to encourage borrowing of items.

During the deployment, we interviewed 10 students about the design and concept of ShareBox. We asked them questions about privacy and sharing within their community.

Iterative Product Design We made changes to the previous ShareBox model (see Figure 2), such as switching from custom-made wooden boxes to metal Ikea lockers and adding a cloud-synced 


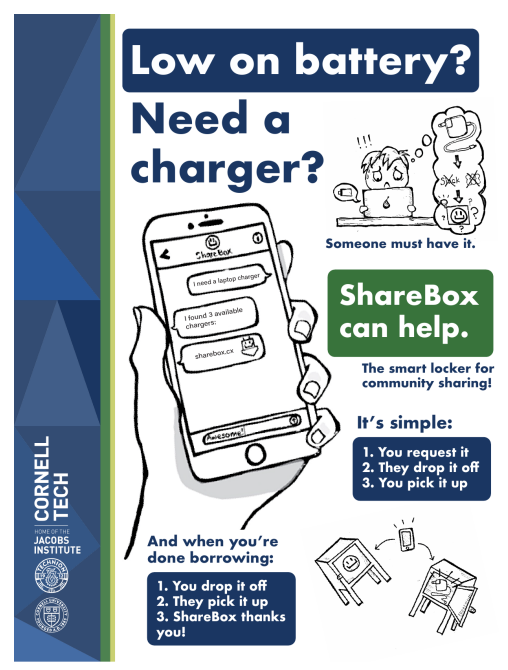

Figure 3: Outreach flyer camera to each box. We made the user path clearer on the website. We also added a new "request" option to allow people to broadcast to the community that they needed an item that was not listed.

\section{CHALLENGES}

We faced significant challenges deploying ShareBox in communities around New York City. First, convincing a local community to host a ShareBox turned out to be difficult, due to two main factors: security concerns and the need for an administrator in the community. After deploying ShareBox, we recognized two more challenges: engaging users and providing a smooth user experience.

Security Concerns Trust and personal safety have been studied in sharing contexts $[2,7,8]$. Designers may want to consider the levels of trust that are present in specific communities before designing a general model. Implementing security measures may be a case-by-case process.

One significant failure was our inability to convince communities outside of our own to adopt our system. Some staff members at these organizations expressed concerns about the system's security. Safeguarding items was a recurring security concern. After we confirmed that there could be multiple items in the box at once, some people voiced worries about theft and suggested we make individual lockers for single items. In response to this concern, we added a camera that would allow the community's administrator to monitor activity through videos taken for each transaction.

Another security concern centered on anonymity, with several people expressing unease about not knowing who was using the box. When we mentioned that we logged users using phone numbers and also had a camera inside the box, this seemed to reassure some but not all of them. At one organization, the staff did not want this feature because they worked with children (this also posed a problem for us, given that our IRB was approved only for adult participants).

However, these concerns about theft and anonymity were less present during the deployment on our campus. Participants expressed high levels of trust in their peers. Some participants even questioned the necessity of certain security features, such as the camera, and pointed out the existing security measures in the building, such as ID card access, cameras in the building, and security guards.

Gatekeeper Issues Designers should consider the burden their models places on administrators.

For each community, our model required an administrator or "champion," a person who would voluntarily assume responsibility for certain aspects of ShareBox, such as user approval, additional promotion, and fielding questions or concerns. It may be that the risk-to-reward ratio was not high enough to convince anyone to volunteer. We tried to recruit people who were already leaders and were enthusiastic about the community. However, we did not offer tangible rewards, such as payment.

A common concern among community leaders was that too much administrative oversight was required of the "champion." One manager at a local organization said that her staff was "already stretched thin" and that adding something else would be too much overhead for her to manage. A shop owner expressed similar concerns. Given the high turnover of her staff, she did not want to 
have to train new employees to be champions. A staff member at a community center also expressed concerns about administrative oversight. She did not want to be responsible for stolen items and did not want there to be any arguments among community members.

User Engagement and Critical Mass Issue Another difficulty we encountered was resolving the tension between security concerns and building a critical mass of users. If we made it easy for users to sign up and access items, e.g., placing the box in a public space and recruiting users across the internet, it might undermine the community's sense of security. Yet, the barriers we placed to access the box may have discouraged people from using it in the first place.

In order to use the box, participants needed to sign up by providing a name and phone number, wait to be approved by the administrator, and then respond to a text chatbot. Only approved users could view the list of available items. To encourage people to sign up, we printed lists of the items and posted them in the common area.

Additionally, anonymity may have acted as a deterrent to user engagement. Originally, anonymity was intended to encourage participation by reducing feelings of indebtedness and removing the ability for users to discriminate based on identity.[4,5] However, several participants and potential hosts at local organizations expressed an interest in knowing the identities of borrowers and lenders. Some also said that they would like to have the ability to thank lenders if they borrowed something. Others noted that they would use the system if they knew their friends were using it.

User Experience and Maintenance Challenges Ideally, a resource exchange system would be easy to use. However, as we mentioned above, the sign-up process delayed immediate user engagement since participants had to wait to be approved. Once participants were approved, the system depended on the use of two interfaces: a browser for listing or requesting items and text messages for coordinating with the box. Additionally, if a participant was logged out, they would need to sign back in by texting "authorize" and their email address, and then checking their email. These layers of interaction may have made the experience less immediate and enjoyable.

We also had to monitor and maintain various features, including the box's wifi-connected lock, the microcontrollers in the box that communicated with the lock and waited for commands based on text messages, the text messaging chatbot service, and the browser-based user interface. Occasionally, wifi or power issues would disable the box, and we would have to reboot or find new power cords.

\section{CONCLUSION}

When designing physical systems to be used by multiple community members, it is important to keep in mind security, staffing, user engagement and ease-of-use.

\section{ACKNOWLEDGMENTS}

This work was supported by the National Science Foundation under Grant No. 1665169. 


\section{REFERENCES}

[1] Mara Balestrini, Paul Marshall, Raymundo Cornejo, Monica Tentori, Jon Bird, and Yvonne Rogers. 2016. Jokebox: Coordinating shared encounters in public spaces. In Proceedings of the 19th ACM Conference on Computer-Supported Cooperative Work \& Social Computing. ACM, 38-49.

[2] Tawanna R Dillahunt and Amelia R Malone. 2015. The promise of the sharing economy among disadvantaged communities. In Proceedings of the 33rd Annual ACM Conference on Human Factors in Computing Systems. ACM, 2285-2294.

[3] Airi Lampinen, Kai Juhani Erkinpoika Huotari, Coye Cheshire, et al. 2015. Challenges to participation in the sharing economy: the case of local online peer-to-peer exchange in a single parents'network. Interaction Design and Architecture (s) (2015).

[4] Airi Lampinen, Vilma Lehtinen, Coye Cheshire, and Emmi Suhonen. 2013. Indebtedness and reciprocity in local online exchange. In Proceedings of the 2013 conference on Computer supported cooperative work. ACM, 661-672.

[5] Matthew V. Law, Mor Naaman, and Nicola Dell. 2018. ShareBox: Designing A Physical System to Support Resource Exchange in Local Communities. In Proceedings of the 2018 Designing Interactive Systems Conference (DIS '18). ACM, New York, NY, USA, 1155-1167. https://doi.org/10.1145/3196709.3196763

[6] Ann Light and Clodagh Miskelly. 2019. Platforms, scales and networks: meshing a local sustainable sharing economy. Computer Supported Cooperative Work (CSCW) (2019), 1-36.

[7] Emily Sun, Ross McLachlan, and Mor Naaman. 2017. TAMIES: a study and model of adoption in P2P resource sharing and indirect exchange systems. In Proceedings of the 2017 ACM conference on Computer supported cooperative work and Social Computing. ACM, 2385-2396.

[8] Jacob Thebault-Spieker, Loren G Terveen, and Brent Hecht. 2015. Avoiding the south side and the suburbs: The geography of mobile crowdsourcing markets. In Proceedings of the 18th ACM Conference on Computer Supported Cooperative Work \& Social Computing. ACM, 265-275. 